\title{
Classificação de Lesões de Pele Utilizando Atributos de Cor e Textura
}

\author{
Nayara H. de Moura ${ }^{1}$, Gabriel C. Moura ${ }^{1}$, Romuere R. V. e Silva ${ }^{1}$, Emerson S. \\ Silva', Rodrigo de M. S. Veras ${ }^{2}$
}

${ }^{1}$ Campus Senador Helvídio Nunes de Barros (CSHNB) - Universidade Federal do Piauí (UFPI) - Picos - PI - Brasil
${ }^{2}$ Departamento de Computação - Universidade Federal do Piauí (UFPI) - Teresina - PI - Brasil
\{naayaraholanda, gabrielmouraufpi, emersilva1003\}@gmail.com,
\{romuere, rveras\}@ufpi.edu.br

\begin{abstract}
Over the past three decades, more people have had skin cancer than all other cancers combined. However, this disease is curable if caught early. This work proposes an analysis of the components of HSV color model by using color and texture features. This extraction was made using medical images of skin lesions. The goal is to promote a classification with a lower error rate. In the tests, we used three classifiers: Support Vector Machine, MultiLayer Perceptron and Random Forest. The experimental results were satisfactory, reaching an accuracy rate of 0.9250 and Kappa index of 0.7541 .
\end{abstract}

Resumo. Ao longo das últimas três décadas, mais pessoas tiveram câncer de pele do que todos os outros tipos de câncer combinados. No entanto, ela é uma doença que tem cura se diagnosticada logo no início. Assim, este trabalho propõe uma análise das componentes do modelo de cores HSV para cor e textura na extração de características. Essa extração é feita através da interpretação de imagens médicas de lesões de pele. Oobjetivo é promover uma classificação com uma menor taxa de erro. Nos testes foram utilizados três classificadores: Máquina de Vetor de Suporte, MultiLayer Perceptron e Random Forest. Os resultados experimentais obtidos foram satisfatórios, atingindo uma acurácia de 0.9250 e um índice Kappa de 0.7541.

\section{Introdução}

O câncer de pele é caracterizado pelas células da pele que multiplicam-se repetidamente até a formação de um tumor maligno. Contudo, é uma doença que tem cura se descoberta logo no início. O melanoma, por exemplo, representa menos de dois por cento dos casos de câncer de pele, mas possui o pior prognóstico e o mais alto índice de mortalidade [American Cancer Society 2015]. De acordo com o Skin Cancer Foundation, em 2015, é estimado que 9.940 pessoas morrerão de melanoma. Além disso, cerca de 3 milhões de câncer de pele não-melanoma e 132.000 melanomas são descobertos a cada ano [World Health Organization 2015].

O diagnóstico no início da doença é essencial. Assim, com a utilização de algoritmos de Processamento Digital de Imagens (PDI), várias técnicas para detecção automática de patologias se concretizaram. O PDI é a manipulação de imagens através de computadores onde a entrada e a saída do processo sejam imagens. Ele tem por objetivo 
o fornecimento de ferramentas para facilitar a identificação e a extração de características encontradas na imagem, para uma posterior classificação [Camara 1996].

Sistemas de diagnóstico automático de lesões cutâneas são ferramentas úteis para a detecção precoce do melanoma. Além disso, proporcionam uma "segunda opinião" ao especialista. Esses sistemas são compostos, em geral, por três etapas: segmentação, extração de características e classificação. O resultado da classificação dependerá de maneira fundamental da qualidade da etapa de extração de características. Dessa forma, para de se obter um melhor resultado na classificação das imagens de lesão de pele são utilizadas as características de cor e textura.

Este trabalho propõe uma análise comparativa para o sistema de cores HSV na extração de características de lesões de pele, através da utilização de suas componentes, tanto de maneira individual, como a soma das mesmas. Para isso, atributos de cor e textura são calculadas sob a área lesionada a partir da utilização desse sistema de cores.

\subsection{Trabalhos Relacionados}

A extração de características descreve a região de interesse da imagem em um formato apropriado para o processamento subsequente, a classificação [Gonzalez e Woods 2010]. No trabalho de Soares (2008), por exemplo, a extração de características é baseada na textura, forma e cor da lesão. A Transformada Wavelet Packet (TWP) é aplicada para extrair a textura, pois consiste em representar a imagem em diferentes bandas de frequência, com distintas resoluções correspondente a cada escala (dilatação e contração). Os atributos de forma são calculados através do descritor de Fourier e de descritores regionais. E os descritores de cor são calculados para toda a imagem e para a lesão a partir da média de seus pixels utilizando os modelos de cores RGB e HSI. Nos testes foram utilizadas 122 imagens, e os resultados obtidos foram promissores. Entretanto, para as lesões não-melanoma, houve uma queda na taxa de acerto.

Conforme Parolin e Herzer (2010), a extração de características foi implementada utilizando a regra $\mathrm{ABCDE}$. A letra $\mathrm{E}$ da regra é utilizada para determinar a Evolução da lesão cutânea. Foram utilizadas 290 imagens (139 não-melanomas e 151 melanomas) de uma base de imagens online para os experimentos. Os resultados encontrados foram pouco satisfatórios, atingindo uma acurácia de $82,55 \%$.

Araújo et al. (2012) propuseram um método de extrair as características da imagem baseado na regra $\mathrm{ABCD}$. A definição da rugosidade é feita a partir da borda extraída, bem como a análise do tamanho da mesma e a quantidade de pontos de inflexão (onde as curvas mudam de côncavas para convexas) presentes. Além disso, o diâmetro, a assimetria e a variação de cores são calculados sob a área lesionada. Este método ainda possui algumas limitações, dentre elas, a pequena quantidade de imagens utilizadas para teste e a sua ineficiência na presença excessiva de pelos.

No trabalho de Cavalcanti et al. (2013) é apresentada uma nova abordagem para a distinção entre melanomas e nevos atípicos (nevos benignos e não usuais, que podem assemelhar-se ao melanoma). $\mathrm{Na}$ diferenciação entre estes dois tipos de lesão de pele, foram utilizadas doze características baseadas na concentração dos dois tipos de melanina, a eumelanina e a feomelanina, em conjunto com a regra $\mathrm{ABCD}$. Neste caso, a característica que pertence à letra $\mathrm{D}$ da regra é alterada para estruturas Diferenciais: rede pigmentar, glóbulos e pontos, que caracterizam lesões melanocíticas. Os experimentos foram feitos com um total de 152 imagens (45 nevos atípicos benignos e 107 melanomas). 
Os resultados obtidos foram animadores, porém, os testes poderiam ter sido feitos com uma base de dados maior.

Este trabalho está organizado da seguinte forma: na Seção 2 são apresentados e descritos os materiais; na Seção 3 é feita uma descrição detalhada do método utilizado; na Seção 4 são expostos os resultados obtidos; e por fim, as conclusões e trabalhos futuros na Seção 5.

\section{Materiais}

Nesta seção, são apresentados e descritos os materiais utilizados nesse trabalho para a extração de características de lesão de pele. Dentre eles, a base de imagens e o sistema de cores.

\subsection{Base de Imagens}

A base de imagens $\mathrm{PH}^{2}$ [Mendonça et al. 2013] é constituída por um total de 200 imagens dermatoscópicas: 80 nevos comuns (manchas), 80 nevos atípicos e 40 melanomas (tumor maligno). Cada imagem possui uma resolução de 768 x 560 pixels no sistema de cores RGB. As cores das peles representadas na base de imagens $\mathrm{PH}^{2}$ podem variar de branco a branco creme.

Um aspecto importante dessa base é que as imagens foram segmentadas e diagnosticadas por um dermatologista. Assim, ela possui um diretório dedicado a cada lesão que contém a imagem dermatoscópica original, a máscara binária da lesão segmentada, bem como a máscara binária das classes de cores presentes na lesão de pele. Esta última, identifica e mostra a localização de cada uma dessas cores. A Figura 1 mostra imagens de lesões de pele pertencentes à base de imagens $\mathrm{PH}^{2}$. As Figuras 1(a) e 1(b), mostram um nevo comum e sua máscara binária da lesão segmentada, respectivamente. As Figuras 1(c) e 1(d) mostram um melanoma e sua máscara binária, respectivamente.

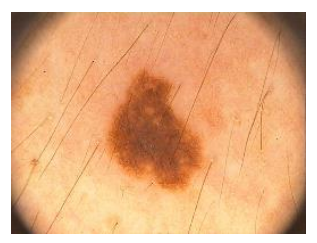

(a)

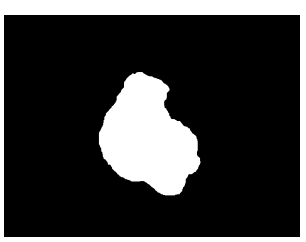

(b)

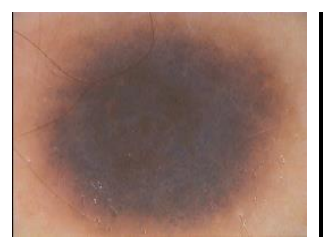

(c)

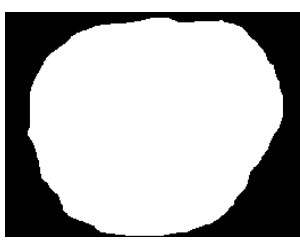

(d)

Figura 1. Imagens de lesões de pele. (a) Nevo comum; (b) Máscara binária da lesão segmentada; (c) Melanoma; (d) Máscara binária da lesão segmentada.

\subsection{Sistema de Cores HSV}

Sistemas de cores são uma representação n-dimensional, onde cada pixel da imagem é representado por um ponto que possui, geralmente, três coordenadas. Seu principal objetivo é a especificação de cores em um formato padronizado.

O sistema de cores HSV é composto pelas componentes: matiz (Hue - H), saturação (Saturation - S) e brilho (Value - V). Essas componentes são responsáveis pela definição do espaço de cor. Além disso, ele é caracterizado por ser uma transformação não-linear do sistema de cores RGB (Red, Green, Blue). A Figura 2 mostra as imagens das componentes do sistema de cores HSV. 


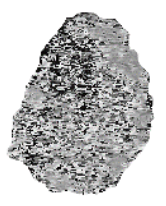

(a)

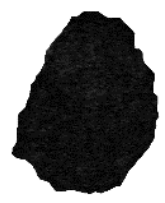

(b)

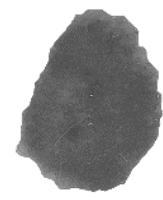

(c)

Figura 2. Componentes do modelo de cores HSV. (a) Componente de matiz; (b) Componente de saturação; (c) Componente de brilho.

O matiz contém a variação de cor de uma imagem, podendo ser vermelho, amarelo ou azul. Seus valores podem variar de 0 a 360. A saturação pode conter valores entre 0 e $100 \%$. Quanto menor este valor, mais cinzenta é a imagem, porém quanto maior, mais saturada ela fica. E por fim, o brilho, que define a luminosidade da cor, que também pode ter valores entre 0 e $100 \%$.

\section{Metodologia}

Nesta seção são descritos os algoritmos utilizados na extração de características de imagens de lesão de pele. A partir da imagem original da lesão e da máscara binária da lesão segmentada, as características de cor e textura são calculadas sob a área lesionada. Tais características são armazenadas em um vetor de atributos para a subsequente classificação dessas imagens em patológica (melanoma) e não-patológica (nãomelanoma). Este processo é repetido para cada componente do sistemas de cores HSV e a soma entre elas. O fluxograma desse método é mostrado na Figura 3.

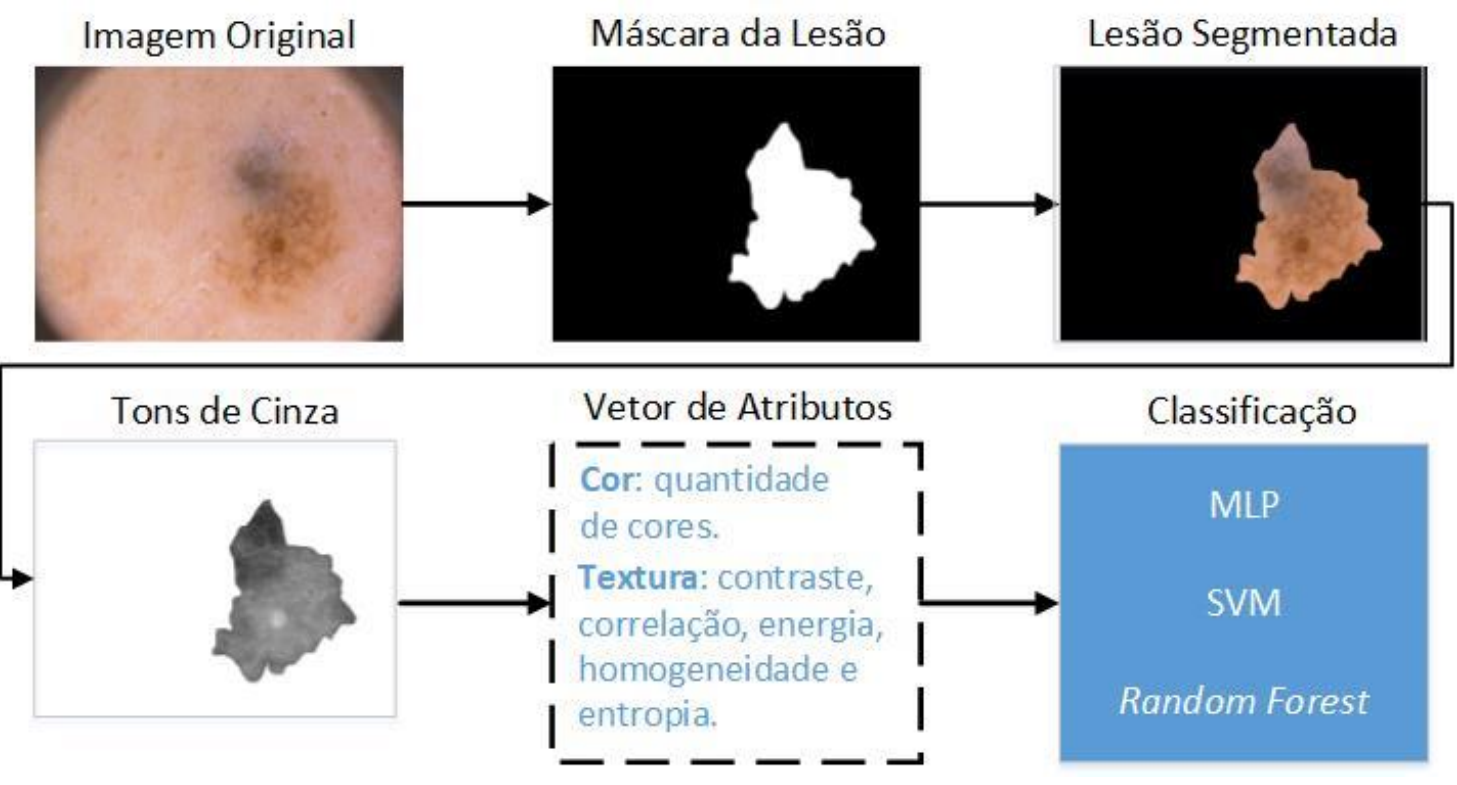

Figura 3. Fluxograma do método.

\subsection{Cor}

O modelo de cores escolhido para o cálculo da variação da coloração interna das lesões foi o HSV. Em cada componente desse modelo, foram feitos testes com cada canal isolado e soma entre eles gerando novas componentes. Um exemplo dessa soma é mostrada na Figura 4. Nesses testes, a quantidade de cores presentes na lesão é utilizada na classificação. Essa quantidade é calculada através da divisão do canal em dez intervalos. 
Desta maneira, as cores pertencentes ao mesmo intervalo foram consideradas semelhantes. A contagem do número de pixels é feita para cada intervalo, sendo descartados os que possuírem regiões muito pequenas (menos de 100 pixels).

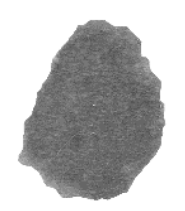

Figura 4. Soma das componentes S e V do modelo de cores HSV.

A Figura 5 mostra um exemplo do cálculo da quantidade de cores presentes na lesão de pele. A coloração foi feita artificialmente através da análise de seus pixels. Nesse exemplo, foram encontradas 5 cores diferentes na lesão. A soma das componentes $\mathrm{S}$ e $\mathrm{V}$ do modelo de cores HSV foi utilizada nesse exemplo.

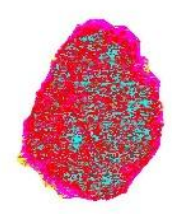

Figura 5. Soma das componentes S e V do modelo de cores HSV. Neste caso, cinco cores foram encontradas.

\subsection{Extração de Atributos via GLCM}

A GLCM (Grey-Level Co-occurrence Matrix) é uma técnica que tem como base a análise de textura em imagens. Nela é analisada as co-ocorrências existentes entre pares de pixels através de algum padrão. Uma GLCM é sempre uma matriz quadrada, onde as informações das intensidades relativas dos pixels são armazenadas. Por este motivo, as imagens utilizadas são sempre em tons de cinza [Haralick et al. 1973].

As probabilidades de co-ocorrências são calculadas entre dois níveis de cinza $i$ e $j$, utilizando uma orientação $\Theta$ (tetha) e uma distância conhecida como pixel pair spacing. Essa orientação pode assumir os valores de $0^{\circ}, 45^{\circ}, 90^{\circ}$ e $135^{\circ}$ [Baraldi e Parmiggiani 1995]. Para cada relacionamento espacial possível (distância e orientação) existe uma matriz de co-ocorrência. Desse modo, todas as informações sobre a textura de uma imagem estarão contidas nessa matriz.

Haralick definiu 14 características significativas para a GLCM. Contudo, a utilização de algumas dessas características podem gerar um melhor desempenho, do que a utilização de todas. Assim, nesse trabalho são feitos cálculos dos seguintes atributos de textura: contraste, correlação, energia, homogeneidade e entropia.

\section{Resultados e Discussões}

$\mathrm{Na}$ análise comparativa dos dados foram utilizadas 200 imagens, dentre estas, 40 melanomas e 160 não-melanomas. Para a classificação das imagens foram utilizados três classificadores: Máquina de Vetor de Suporte (SVM) [Haykin 2008], MultiLayer 
Perceptron (MLP) [Haykin 2008] e Random Forest [Breiman 2001]. O método estatístico $k$-fold cross-validation $(k=10)$ foi utilizado para gerar os resultados.

Os resultados foram analisados de acordo com o índice Kappa (K) [Rosenfield e Fitzpatrick-lins 1986], a área sob a curva ROC (AUC) e a Acurácia (A). Além disso, os experimentos foram realizados para as características de cor e textura com cada componente do modelo de cores HSV e a soma entre elas. Essa soma é representada pelo símbolo "+" nas Tabelas 2, 3 e 4. O Verdadeiro Positivo (VP) e o Verdadeiro Negativo (VN) apresentam a quantidade de lesões não-melanocíticas e melanocíticas, respectivamente, classificadas corretamente, já o Falso Positivo (FP) e o Falso Negativo (FN) as classificadas incorretamente.

O nível de precisão do índice Kappa utilizado para a avaliação dos resultados é mostrada na Tabela 1.

Tabela 1. Nível de precisão da classificação, de acordo com o índice Kappa.

\begin{tabular}{c|c}
\hline Índice Kappa $(\mathrm{K})$ & Qualidade \\
\hline $\mathrm{K} \leq 0.2$ & Ruim \\
\hline $0.2<\mathrm{K} \leq 0.4$ & Razoável \\
\hline $0.4<\mathrm{K} \leq 0.6$ & Bom \\
\hline $0.6<\mathrm{K}<0.8$ & Muito Bom \\
\hline $\mathrm{K} \geq 0.8$ & Excelente \\
\hline
\end{tabular}

Os resultados encontrados com a utilização do classificador $M L P$ são mostrados na Tabela 3. A soma das bandas $\mathrm{S}$ e V do modelo de cores HSV também obtiveram um melhor resultado atingindo uma acurácia de 0,9250 e um índice Kappa de 0,7541. No entanto, com o classificador Random Forest, apresentado na Tabela 4, o melhor resultado é alcançado com a utilização da banda $\mathrm{H}$, que obteve uma acurácia de 0,8900 e um índice Kappa de 0,6497.

Tabela 2. Resultados para o classificador SVM.

\begin{tabular}{c|c|c|c|c|c|c|c}
\hline & VP & FP & FN & VN & K & AUC & A \\
\hline$H$ & 152 & 8 & 14 & 26 & 0,6358 & 0,8000 & 0,8900 \\
\hline $\mathrm{S}$ & 155 & 5 & 16 & 24 & 0,6341 & 0,7840 & 0,8950 \\
\hline $\mathrm{V}$ & 156 & 4 & 15 & 25 & 0,6690 & 0,8000 & 0,9050 \\
\hline $\mathrm{H}+\mathrm{S}$ & 153 & 7 & 13 & 27 & 0,6689 & 0,8160 & 0,9000 \\
\hline $\mathrm{H}+\mathrm{V}$ & 156 & 4 & 24 & 16 & 0,4615 & 0,6880 & 0,8600 \\
\hline $\mathbf{S}+\mathrm{V}$ & $\mathbf{1 5 6}$ & $\mathbf{4}$ & $\mathbf{1 4}$ & $\mathbf{2 6}$ & $\mathbf{0 , 6 8 9 7}$ & $\mathbf{0 , 8 1 3 0}$ & $\mathbf{0 , 9 1 0 0}$ \\
\hline
\end{tabular}

Tabela 3. Resultados para o classificador MultiLayer Perceptron.

\begin{tabular}{c|c|c|c|c|c|c|c}
\hline & VP & FP & FN & VN & K & AUC & A \\
\hline$H$ & 151 & 9 & 10 & 30 & 0,7003 & 0,9200 & 0,9050 \\
\hline $\mathrm{S}$ & 150 & 10 & 13 & 27 & 0,6302 & 0,8970 & 0,8850 \\
\hline $\mathrm{V}$ & 152 & 8 & 16 & 24 & 0,5946 & 0,9030 & 0,8800 \\
\hline $\mathrm{H}+\mathrm{S}$ & 150 & 10 & 14 & 26 & 0,6104 & 0,9100 & 0,8800 \\
\hline $\mathrm{H}+\mathrm{V}$ & 149 & 11 & 16 & 24 & 0,5574 & 0,8670 & 0,8650 \\
\hline $\mathbf{S}+\mathbf{V}$ & $\mathbf{1 5 5}$ & $\mathbf{5}$ & $\mathbf{1 0}$ & $\mathbf{3 0}$ & $\mathbf{0 , 7 5 4 1}$ & $\mathbf{0 , 9 3 8 0}$ & $\mathbf{0 , 9 2 5 0}$ \\
\hline
\end{tabular}

Tabela 4. Resultados para o classificador Random Forest. 


\begin{tabular}{c|c|c|c|c|c|c|c}
\hline & VP & FP & FN & VN & K & AUC & A \\
\hline H & $\mathbf{1 5 0}$ & $\mathbf{1 0}$ & $\mathbf{1 2}$ & $\mathbf{2 8}$ & $\mathbf{0 , 6 4 9 7}$ & $\mathbf{0 , 9 1 4 0}$ & $\mathbf{0 , 8 9 0 0}$ \\
\hline $\mathrm{S}$ & 150 & 10 & 12 & 28 & 0,4970 & 0,9070 & 0,8900 \\
\hline $\mathrm{V}$ & 154 & 6 & 15 & 25 & 0,6416 & 0,8970 & 0,8950 \\
\hline $\mathrm{H}+\mathrm{S}$ & 150 & 10 & 12 & 28 & 0,6497 & 0,8990 & 0,8900 \\
\hline $\mathrm{H}+\mathrm{V}$ & 150 & 10 & 17 & 23 & 0,5485 & 0,8690 & 0,8650 \\
\hline $\mathrm{S}+\mathrm{V}$ & 150 & 10 & 14 & 26 & 0,6104 & 0,9080 & 0,8800 \\
\hline
\end{tabular}

Os três classificadores alcançaram resultados satisfatórios, porém, o MLP se destacou. Além disso, pode ser observado que a componente gerada pela soma das bandas $\mathrm{S}$ e V do modelo de cores HSV obteve maior sucesso para extração de atributos em lesões de pele. Ela apresentou uma acurácia de 0,9250 e um índice Kappa igual a 0,7541 na classificicação.

\section{Conclusão e Trabalhos Futuros}

Este trabalho mostrou a extração de características de lesões de pele utilizando as características de cor e textura. Para isso, os atributos foram testados com as diferentes bandas do modelo de cor HSV e das combinações entre elas, a fim de encontrar a melhor classificação. O melhor resultado obtido foi um índice Kappa de 0,7541 encontrado através da utilizando a soma das bandas $\mathrm{S}$ e V. A partir da análise da Tabela 1 e do valor do índice Kappa apresentado na Tabela 3 foi possível concluir que o método se mostrou eficiente, obtendo um desempenho "Muito Bom".

A principal contribuição desse trabalho é a análise dos descritores de cor e textura no modelo de cores HSV, a fim de melhorar a extração de características em imagens de lesão de pele. Nesse sentido, a utilização desses descritores proporcionaram um aumento na taxa de acerto e, consequentemente, no índice Kappa durante a classificação das imagens.

No subsequente desenvolvimento desse projeto almeja-se utilizar outros descritores para a extração de atributos de lesões de pele, tais como: GLRLM, LBP, HOG SURF, FOURIER e HAAR. Além disso, máquinas de comitê serão utilizadas na etapa de classificação das imagens. Serão aplicados outros sistemas de cores em comparação ao HSV, sendo eles: HSI, L* $u^{*} v$ e CMYK. E finalmente, a comparação dos resultados obtidos com o estado da arte.

\section{Referências}

American Cancer Society (2015) "Cancer facts \& figures 2015", http//www.cancer.org/acs/groups/content/@editorial/documents/document/acspc044552.pdf, abril.

Araújo, A. F. et al. (2012) "Análise e Caracterização de Lesões de Pele para Auxilio ao Diagnóstico Médico”. In Neves, L. A. P., Neto, H. V. e Gonzaga, A. (Eds.). Avanços em Visão Computacional. Paraná: omnipax. p. 27-46.

Baraldi, A. e Parmiggiani, F. (1995) "An investigation of the textural characteristics associated with gray level cooccurrence matrix statistical parameters". Geoscience and Remote Sensing, IEEE Transactions on, v. 33, n. 2, p. 293-304.

Breiman, L. (2001) "Random forests". Machine Learning, v. 45, n. 1, p. 5-32. 
Camara, G. et al. (1996) "SPRING: Integrating remote sensing and GIS by objectoriented data modelling". Computers \& Graphics, v. 20, n. 3, p. 395-403.

Cavalcanti, P. G., Scharcanski, J. e Baranoski, G. V. G. (2013) "A two-stage approach for discriminating melanocytic skin lesions using standard cameras". Expert Systems with Applications, v. 40, n. 10, p. 4054-4064.

Gonzalez, R. C. e Woods, R. E. (2010) "Processamento Digital de Imagens". $3^{\mathrm{a}}$ ed. São Paulo: Pearson Prentice Hall, 640 p.

Haralick, R., Shanmugam, K. e Dinstein, I. (1973) "Textural features for image classification". Systems, Man and Cybernetics, IEEE Transactions on, n. 6, p. 610621.

Haykin, S. (2008) 'Neural Networks and Learning Machines". 3 $3^{\mathrm{a}}$ ed. New Jersey: Prentice Hall, 936 p.

Mendonça, T. et al. (2013) " $\mathrm{PH}^{2}$ - A dermoscopic image database for research and benchmarking". In Engineering in Medicine and Biology Society (EMBC). 35th Annual International Conference of the IEEE. Osaka, Japan, p. 5437-5440.

Parolin, A., Herzer, E. e Jung C. R. (2010) "Semi-automated diagnosis of melanoma through the analysis of dermatological images". In Graphics, Patterns and Images (SIBGRAPI), 2010 23rd SIBGRAPI Conference on. IEEE, p. 71-78.

Rosenfield, G. H. e Fitzpatrick-lins, K. A. (1986) "A coefficient of agreement as a measure of thematic classification accuracy". Photogrammetric Engineering and Remote Sensing, v. 52, p. 223-227.

Skin Cancer Foundation (2015) "Skin cancer facts", http://www.skincancer.org/skincancer-information/skin-cancer-facts\#melanoma, abril.

Soares, H. B. (2008) "Análise e classificação de imagens de lesões da pele por atributos de cor, forma e textura utilizando máquina de vetor de suporte". 180 f. Tese (Doutorado em Engenharia Elétrica) - Universidade Federal do Rio Grande do Norte.

World Health Organization (2015) “How common is skin cancer?", http//www.who.int/uv/faq/skincancer/en/index1.html, abril. 